\title{
In Memory of the Late Professor Hatsujiro Hashimoto: Toward the Realization of His Dream
}

\author{
Yoshizo Takai
}

Department of Material and Life Science, Graduate School of Engineering, Osaka University, Suita, Osaka, Japan

Professor Hatsujiro Hashimoto, the $9^{\text {th }}$ president of the IFSEM and a distinguished scientist in the Microscopy Society of America, passed away on April 15 th 2017 at the age of 95. I would like to express my heartfelt sorrow on the death of this great scientist and educator.

Professor Hashimoto was born in the Shiga prefecture on December $26^{\text {th }}, 1921$. He studied physics at the Hiroshima University, where he experienced the atomic bomb explosion at a very close distance but miraculously escaped certain death, and he devoted the rest of his life toward research and education. After graduating from the University in September 1945, he began to conduct research at the Kyoto University and started to think about the importance of electron diffraction studies combined with electron microscopy observations. He successfully constructed a three-lens electron microscope in 1953 [1] based on a previous work (Fig. 1). It was his firm belief that new discoveries come along with the development of new experimental equipment. Professor Hashimoto worked at the Kyoto Technical University from 1950 to 1972, the Osaka University from 1972 to 1985, and the Okayama University of Science from 1985 to 2001. During a period of over a half a century, he observed dislocations by Moire patterns [2], pioneered studies of environmental TEM to observe the dynamic processes associated with crystal growth [3], characterized the anomalous absorption effect in dynamic electron diffraction [4], imaged single atoms by high-resolution dark-field imaging [5], developed a $400 \mathrm{kV} \mathrm{HRTEM}$ and conducted dynamic observations of the movement of atoms [6], and executed energy-filtered TEM imaging [7]. In his time at the Osaka University, he was particularly focused on conducting HRTEM observations of various phenomena in the field of materials science. The ultimate goal of his studies was to visualize and understand chemical and physical phenomena such as the movement of individual atoms. Although the TEM resolution at that time was not high enough to resolve individual atoms, he managed to observe the movement of atoms around defects using a TV recording system [6]. Although this TV recording system made dynamic observations using environmental TEM relatively easy, it was difficult to precisely determine the positions of atoms because of the residual spherical aberration.

To realize Professor Hashimoto's dream, we, his students, discussed and reached a conclusion that the spherical aberration must first be corrected. One of his students developed an image-processing method called defocus image-modulation processing (DIMP) [8] to correct the effect of the spherical aberration using the two techniques: high-speed focus modulation and weighted image integration by controlling the exposure time. By this processing, the spherical aberration could be corrected at a video rate $(1 / 30 \mathrm{~s})$ [9]. However, it is still difficult to keep the position and focus constant on an atomic scale as the environmental conditions vary. Now, we are working to develop a new version of this tool, a real time wave field reconstruction TEM system that enables atomic-scale dynamic analyses while performing auto-focus tracking and correction of coma aberration and two-fold and three-fold astigmatisms at a video rate $(1 / 30 \mathrm{~s})$ [10]. In the developed system (Fig. 2), a high-speed image processing technique running on a graphical processing unit is newly incorporated to calculate the exit wave field from the original wave field obtained experimentally by the defocus image-modulation processing method. 
References:

[1] H Hashimoto, J. Phys. Soc. Jpn. 9 (1954) p. 150.

[2] H Hashimoto, R Uyeda, Acta Cryst. 10 (1957) p.143.

[3] H Hashimoto, K Tanaka and E Yoda, J. Electron Microsc. 6 (1958) p. 8.

[4] H Hashimoto, A Howie, MJ Whelan et al., Proc. R. Soc. A269 (1962) p. 80.

[5] H Hashimoto, A Kumao, K Hino et al., Jpn. J. Appl. Phys. 10 (1973) p. 1115.

[6] H Hashimoto, Y Takai, Y Yokota et al., Jpn. J. Appl. Phys. 19 (1980) p. L1.

[7] N Ajika, H Hashimoto, H Endoh et al., J. Electron Microsc. 32 (1983) p. 250.

[8] T Ikuta, J. Electron Microsc., 38 (1989) p. 415.

[9] Y Takai, T Kawasaki, Y Kimura et al., Phys. Rev. Lett. 87 (2001) p. 106105.

[10] T Tamura, Y Kimura and Y Takai, J. Electron Microsc. 66 (2017) p. 172.
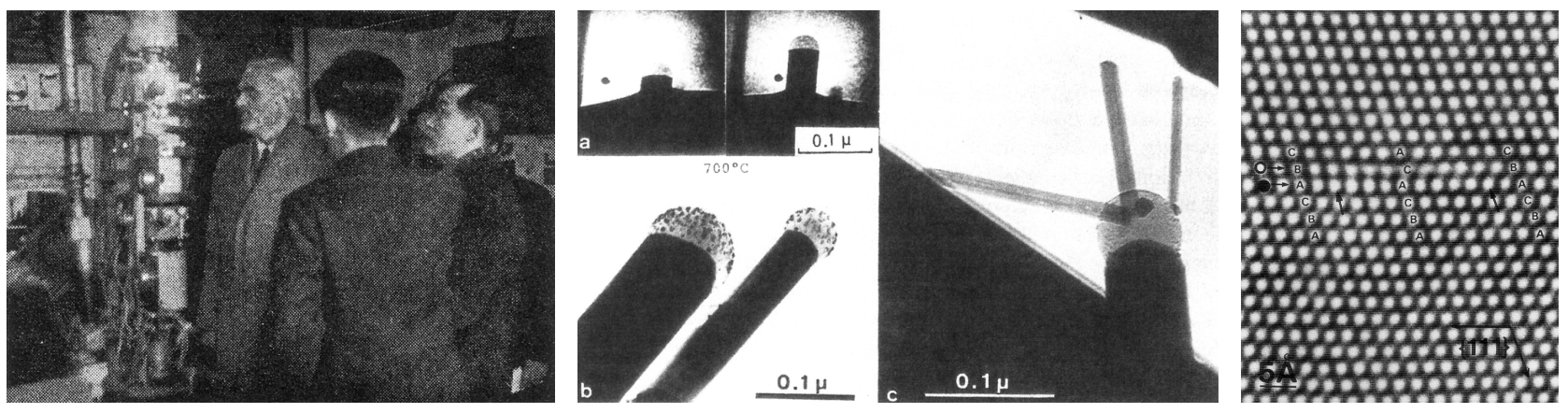

Figure 1. (a) Universal TEM [1], (b) tungsten-oxide needle growth [3], and (c) Au crystal defect [6].

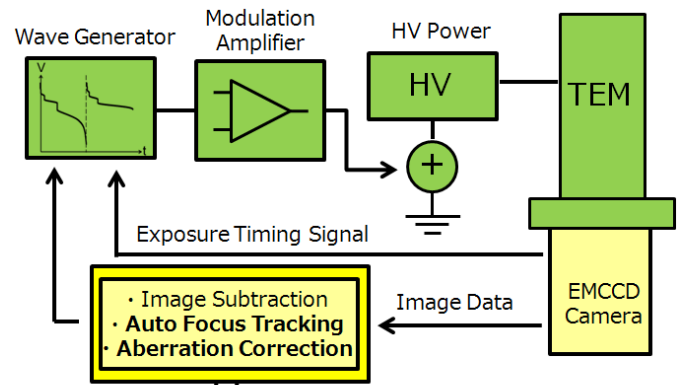

(a)

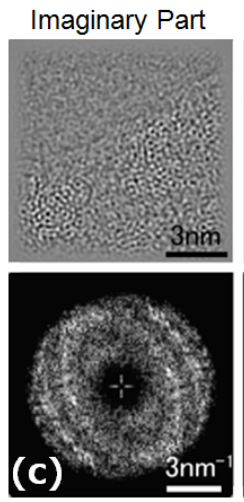

Real Part

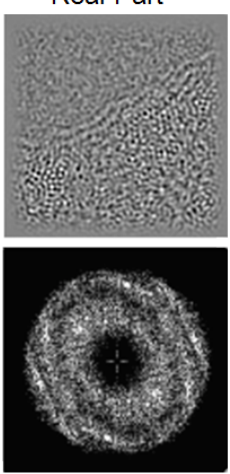

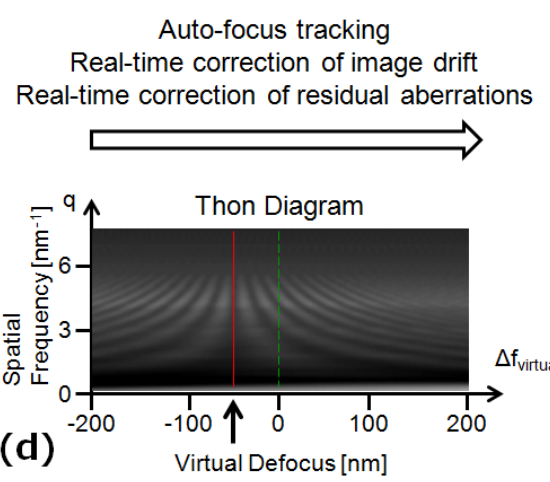

Auto-focus tracking Real-time correction of image drift Real-time correction of residual aberrations

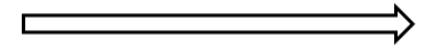

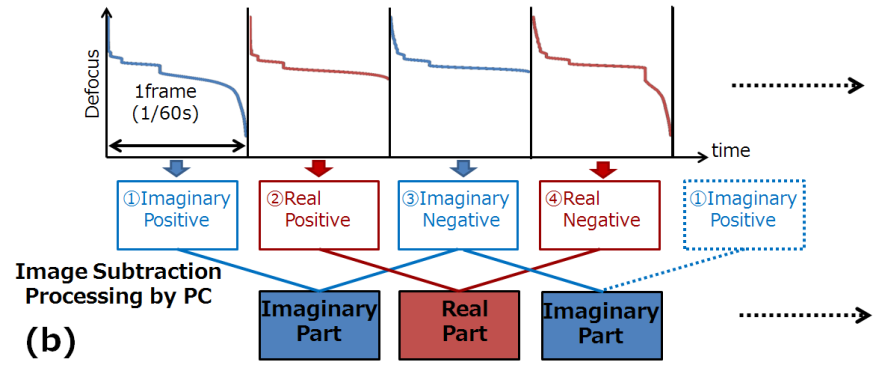

Imaginary Part
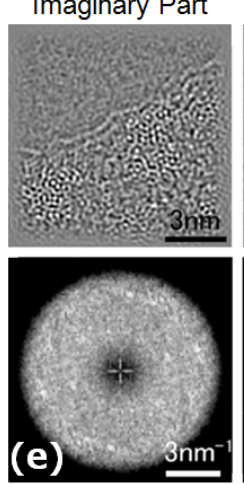

Real Part

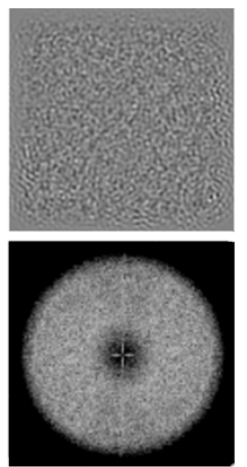

Figure 2. (a) Schematic of the real-time wave-field-reconstruction TEM system with (b) a timing chart. (c) Original wave field reconstructed by the real-time DIMP, (d) Thon diagram, and (e) the final wave field obtained after correcting the phase shift induced by the virtual defocus and residual aberrations. [10] 